\title{
CONTRIBUTION-BASED PEER SELECTION FOR PACKET PROTECTION FOR P2P VIDEO STREAMING OVER MESH-BASED NETWORKS
}

\author{
Chi-Wen Lo ${ }^{1}$, Chia-Wen Lin ${ }^{1}$, Yung-Chang Chen ${ }^{1}$, and Jen-Yu Yu ${ }^{2}$ \\ ${ }^{1}$ Department of Electrical Engineering, National Tsing Hua University, Hsinchu, Taiwan \\ ${ }^{2}$ Information and Communications Research Labs, Industrial Technology Research Institute, Taiwan
}

\begin{abstract}
This paper proposes a distributed packet protection mechanism that can minimize the packet loss probability for mesh based P2P video streaming systems. The proposed scheme combines a peer selection method with forward error correction (FEC) codes. The parent peers select the child peers, which can achieve the minimal packet loss probability compared to other candidate child peers, to transmit the FEC redundant substream. Moreover, the proposed scheme utilizes a packet loss model to estimate the packet loss probability in a mesh based $\mathrm{P} 2 \mathrm{P}$ network. The packet loss propagation among peers is modeled through Markov random field (MRF). Simulation results demonstrate that our scheme can effectively mitigate packet loss in a mesh-based P2P network.
\end{abstract}

Index Terms - Error protection, P2P video streaming system, peer selection, FEC

\section{INTRODUCTION}

Due to the rapid growth of advanced network and multimedia technologies, video streaming services are able to provide stable quality. The key of a successful video streaming system lies in the video quality in the user watching experiences. However, one of the major challenges to video streaming services is packet loss. If a video packet cannot be received before its playback time, the reconstructed video quality may be seriously damaged.

Packet-level FEC has been often utilized to protect video packets in video streaming systems, where the channel encoder, such as Reed-Solomon code, encodes the video bitstreams into $k$ data packets and extra $n-k$ redundant packets, denoted as $F E C(n, k)$. The receiver can fully reconstruct the original signal if at least any $k$ out of $n$ packets are received. On the other hand, $F E C(n, k)$ scheme can tolerate $n-k$ packets loss at most. The FEC protection capability can be increased by increasing $n$ to ensure enough packets can be received. Compared with retransmission-based schemes in which the receiver requests the re-transmissions for the lost packets, the FEC-based scheme is more suitable for timesensitive video applications, such as real-time video streaming.

The method proposed in [1] applies FEC to recover packet loss in an overlay streaming system. The FEC codes are decided according to the channel conditions of the segments of a delivery path but the peer dynamics is not considered. The performance of FEC codes with different video frame types was analyzed in [2]. Due to the motion prediction structure of video encoder, I and P frames are more important than B frames. The results in [2] show that the packet loss probability in the $\mathrm{P} 2 \mathrm{P}$ video streaming system can be reduced by unequal error protection (UEP). With UEP, the important video frames are assigned to more redundancy to overcome packet loss. In [3], the packet loss probability and loss accumulation in a multi-source tree-based P2P system are analyzed.

However, FEC based schemes consume more bandwidth resource to transmit the redundant packets. In the current network environments, the down-link bandwidth of peers is sufficient to receive the real-time video streaming, but the up-link bandwidth of peers cannot be satisfied the substream demands from other peers. If the available up-link bandwidth of a P2P system cannot afford the enormous amount of redundant packets, packets will be dropped due to traffic congestion. Therefore, the limited up-link bandwidth must be utilized efficiently. Besides, the demands of redundant packets vary largely for the heterogeneous peers. An adaptive data protection method to choose the appropriate amounts of redundant packets for each peer should be addressed.

In this paper, we propose a sender-driven approach by which a parent-peer can adaptively select child-peers to transmit redundant packets according to the packet loss rate, peer dynamics condition, and packet loss propagation among peers. An analytic model was proposed in [3] to estimate the packet loss probability of candidate child-peers in tree structures. However, the model cannot be applied to mesh structures. The error propagation of P2P video streaming in a mesh-based network was rarely addressed because of the network's irregular structure. We analyze the packet loss propagation for the mesh network and use Markov random field (MRF) to model the interrelation among peers. By using the MRF model, each parent peer can optimally assign the redundant packets to child peers under the uplink bandwidth constraint.

The rest of this paper is organized as follows. In Section 2, we present a framework of FEC-based error protection for push-pull $\mathrm{P} 2 \mathrm{P}$ video streaming. The proposed contribution-guided peer selection mechanism is described in Section 3. Section 4 shows the simulation results. Finally, conclusions are drawn in Section 5.

\section{FEC-BASED ERROR PROTECTION FOR P2P VIDEO STREAMING}

Fig. 1 depicts our packetization method. Suppose each group of pictures (GOP) is encoded with the same bitrate and with the same number of video packets. Then, the $k$ video source packets of each GOP are encoded with $F E C(n, k)$ code to generate additional $n-k$ redundant packets. The corresponding packets of the GOPs compose the video substreams. As shown in Fig. 1, video substream 1 contains the first packets from GOP \#1 through GOP $\# N$. During a streaming session, the child-peers request their parent-peers for the video substreams (i.e., a pull process). Once the parent-peers accept the requests, the parent-peers continuously push the corresponding packets to their child peers, as known as the push-pull methods [4]. 


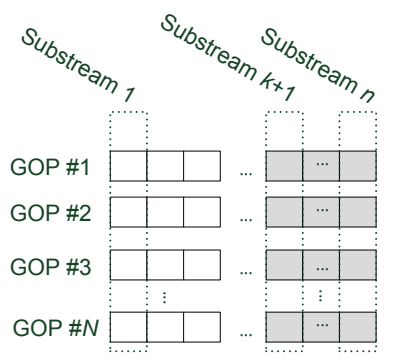

Fig. 1. Data protection with $F E C(n, k)$, where white blocks indicate the data packets and the grey ones indicate FEC redundant blocks.

When a new peer joins the P2P system, it starts contacting the bootstrap server to obtain the live peer list. Then the new peer requests at least $k$ data substreams from the peers in the live peer list. Besides the $k$ data substreams, the peer also requests additional redundant substreams to reduce the possibility of packet loss during video transmissions by sending the request to parent-peer $x$ that can offer the redundant substreams, and is added into the candidate set $\overline{\mathbf{C}}_{x}$ of peer $x$. If the uplink capacity of peer $x$ cannot afford the demands of all peers in $\overline{\mathbf{C}}_{x}$, those "low-contribution" candidate peers will be rejected. The contribution of a candidate peer is measured by the energy function defined in (12).

When peer $y$ obtains $r_{y}$ redundant substreams, and requests one more redundant substream from peer $x$. The total number of substreams expected to be received by peer $y$ will be $n_{y}=k+r_{y}+$ $\sigma_{x y}$, where $\sigma_{x y}$ is the activity of the link between parent-peer $x$ and candidate child-peer $y$. If peer $x$ accepts peer $y$ as its child-peer, $\sigma_{x y}$ $=1$; otherwise, $\sigma_{x y}=0$. We use the set of random variable $\bar{\sigma}=$ $\left\{\sigma_{x y} \mid x \in \overline{\boldsymbol{L}}, y \in \overline{\boldsymbol{C}}_{x}\right\}$ to represent the topological configuration among peers, where $\overline{\boldsymbol{L}}$ denotes the set of live peers. The configuration constitutes a topology that describes the interrelation of packet transport among peers.

\section{PROPOSED PEER SELECTION SCHEME}

We investigate the peer interrelations in mesh networks in terms of packet loss probability and propose a scheme for finding the optimal configuration that can minimize packet loss probability.

\subsection{The Packet Loss Models}

The packet loss models proposed in [3] can be used to estimate the packet loss probability of the peers at depth $D$ in a tree, denoted as $Q_{D}$. In this model, the packet loss caused by the following four events are taken into considerations: 1) peer arrival/departure behavior described by a continuous-time Markov chain (CTMC), 2) FEC coding pattern, 3) packet drop rate of the channel, and 4) packet loss probability of the parent-peers at depth $D-1$. These events are denoted as $P_{i}, F E C(n, k), d$, and $Q_{D-1}$, respectively. However, unlike the case in a tree-based network, peers in a meshbased network do not belong to specific levels/depths. Furthermore, each peer suffers from different packet loss conditions. Thus, the derivation of packet loss models of a peer for a mesh-based network is much more complex. In what follows, we briefly summarize the tree- based packet loss models derived in [3].

The packet loss probability of candidate peer $y$ that has received $r_{y}$ substreams and requests one more redundant substream from peer $x$ can be formulated as [3]

$$
Q_{y}\left(r_{y}+\sigma_{x y}\right)=\sum_{i=0}^{n_{y}} P_{i} q_{y, i}
$$

where $P_{i}$ is the probability of $i$ parent-peers leaving, which can be modeled by CTMC [3], and $q_{y, i}$ is the packet loss probability due to the leaving of $i$ parent-peers. The calculation of $q_{y, i}$ is described below.

When the number of leaving parent-peers exceeds $r_{y}+\sigma_{x y}$ (i.e., $i>r_{y}+\sigma_{x y}$ ), the lost packets cannot be recovered. Therefore, a packet is lost due to either of the three events: 1) the number of parent-peers leaving the system exceeds $\left.r_{y}+\sigma_{x y}, 2\right)$ the live parent-peers do not have the packet, and 3) the packet is dropped during the transmission. These conditions are expressed by

$$
q_{y, i>r_{y}+\sigma_{x y}}=\frac{i}{n_{y}}+\left(\frac{n_{y}-i}{n_{y}} \cdot Q_{y}^{n}\right)+\left(\frac{n_{y}-i}{n_{y}}\right) \cdot\left(1-Q_{y}^{n}\right) \cdot d_{y}
$$

where the three terms on the right-hand-side of (2) represent the occurrence probabilities of the three events, respectively. In (2), $Q_{y}^{n}$ is the average packet loss probability of the parent-peers of peer $y$ as calculated by

$$
Q_{y}^{n}=\frac{1}{k+r_{y}} \sum_{p \in \operatorname{parent}(y)} Q_{p},
$$

where parent $(y)$ is the set of parent peers of peer $y . d_{y}$ denotes the average channel packet drop rate between peer $y$ and its parentpeers as follows:

$$
d_{y}=\frac{1}{k+r_{y}} \sum_{p \in \operatorname{parent}(y)} d_{y, p}
$$

As shown in (2), when the number of leaving peers $i \leq n_{y}-k$, the lost packets may be recovered. However, the lost packets caused by the parent-peer departure are not recoverable, should the number of received packets from the $n_{y}-i$ surviving live peers be less than $k$. This packet loss probability $C_{1}$ can be calculated by

$$
\begin{array}{r}
C_{1}=\frac{i}{n_{y}} \cdot\left\{\sum_{w=0}^{k-1}\left(\begin{array}{c}
n_{y}-i \\
\mathrm{w}
\end{array}\right)\left[\left(1-Q_{y}^{n}\right)\left(1-d_{y}\right)\right]^{w}[1\right. \\
\left.\left.-\left(1-Q_{y}^{n}\right)\left(1-d_{y}\right)\right]^{n_{y}-i-w}\right\}
\end{array}
$$

Besides, a lost packet due to the event that the live parent peer does not have the packet is not recoverable, if the number of received packets from the surviving live peers other than peer $y$ is less than $k$. This packet loss probability $C_{2}$ can be calculated by

$$
\left.\begin{array}{rl}
C_{2}=\left(\frac{n_{y}-i}{n_{y}} \cdot Q_{y}^{n}\right) & \cdot\left\{\sum_{w=0}^{k-1}\left(\begin{array}{c}
n_{y}-i-1 \\
\mathrm{w}
\end{array}\right)\left[\left(1-Q_{y}^{n}\right)\left(1-d_{y}\right)\right]^{w}[1\right. \\
& \left.-\left(1-Q_{y}^{n}\right)\left(1-d_{y}\right)\right]^{n_{y}-i-1-w}
\end{array}\right\}
$$

Finally, a lost packet that gets dropped during the transmission cannot be recovered, if the number of received packets from the surviving live peers other than peer $y$ is less than $k$. This loss probability $C_{3}$ can be calculated by

$$
\begin{aligned}
C_{3}=\left\{\frac{n_{y}-i}{n_{y}} \cdot(1-\right. & \left.\left.Q_{y}^{n}\right) \cdot d_{y}\right\} \\
& \cdot\left\{\sum_{w=0}^{k-1}\left(\begin{array}{c}
n_{y}-i-1 \\
\mathrm{w}
\end{array}\right)\left[\left(1-Q_{y}^{n}\right)\left(1-d_{y}\right)\right]^{w}[1\right. \\
& \left.\left.-\left(1-Q_{y}^{n}\right)\left(1-d_{y}\right)\right]^{n_{y}-i-1-w}\right\}
\end{aligned}
$$


As a result, $q_{y, i \leq r_{y}+\sigma_{y}}$ is obtained by

$$
q_{y, i \leq r_{y}+\sigma_{x y}}=C_{1}+C_{2}+C_{3}
$$

\subsection{Estimation of a Candidate Peer's Contribution}

In the proposed peer selection scheme, when a candidate peer requests a redundant substream from a parent-peer, the parent-peer will evaluate the candidate peer's contribution of forwarding the substream to its succeeding child-peers that also request the substream. To this end, we estimate the packet loss reduction contributed by a candidate peer. The more the packet loss reduction, the higher the candidate peer's contribution. Since peer $y$ is also a parent-peer, the packet loss occurring in peer $y$ will propagate to its child-peers as well. When peer $y$ obtains one additional redundant substream, the influence of packet loss reduction $\Delta Q_{y}$ on the average packet loss probability of a childpeer $z$ can be estimated by

$$
Q_{z}^{n}=\frac{1}{k+r_{z}}\left[\left(\sum_{p \in \text { parent(z) }} Q_{p}\right)-\Delta Q_{y}\right],
$$

where

$$
\Delta Q_{y}=Q_{y}\left(r_{y}\right)-Q_{y}\left(r_{y}+1\right) .
$$

Thus, the packet loss reduction (contributed by peer $y$ ) for peer $z$ can be calculated by

$$
\Delta Q_{z}=Q_{z}\left(r_{z}\right)-Q_{z}\left(r_{z}, \Delta Q_{y}\right)
$$

where $Q_{z}\left(r_{z}, \Delta Q_{y}\right)$ is the packet loss probability of peer $z$ that receives $r_{z}$ redundant substreams which is a function of $\Delta Q_{y}$ as in (9). Then, $\Delta Q_{z}$ can influence the next-level child-peers in the same way. As a result, such packet loss reduction contributed by a peer would benefit the peer's succeeding child-peers of different levels.

\subsection{Contribution-Guided Peer Selection}

In our peer selection process, parent-peer $x$ selects the candidate peers in $\overline{\mathbf{C}}_{x}$ to transmit the redundant substreams according to the candidate peers' contribution in assisting the delivery of video data. However, in a mesh-based network, it is difficult to model the interrelation among peers since the topology of interconnection is complex, making the estimation of a peer's contribution itself a very challenging problem. We propose to model the interrelation between peers in a mesh-based network using MRF [5] to address the problem. In our peer selection method, the network topology is determined according to the configuration of packet request, $\bar{\sigma}$. The energy function of the configuration is defined as follows:

$$
\begin{gathered}
H(\bar{\sigma} \mid \bar{Q}, \bar{r})=H_{0}(\bar{\sigma} \mid \bar{Q}, \bar{r})+\cdots+H_{M}(\bar{\sigma} \mid \bar{Q}, \bar{r}) \\
H_{0}(\bar{\sigma} \mid \bar{Q}, \bar{r})=\sum_{y \in \bar{C}_{x}} Q_{y}\left(r_{y}+\sigma_{x y}\right) \\
H_{1}(\bar{\sigma} \mid \bar{Q}, \bar{r})=\sum_{y \in \overline{\boldsymbol{C}}_{x}} \sum_{z \in \operatorname{child}(y)} Q_{z}\left(r_{z}, \Delta Q_{y}\right)
\end{gathered}
$$

where $\bar{Q}=\left\{Q_{l} \mid l \in \overline{\boldsymbol{L}}\right\}$ denotes the set of packet loss probabilities of peers, $\left.\bar{r}=\left\{r_{l} \mid l \in \bar{L}\right\}\right\}$ is the set of the numbers of peers' received redundant substreams. In (12), the energy includes the packet loss probability of the peers in $\overline{\boldsymbol{C}}_{x}$, denoted as $H_{o}$, and the packet loss probability of the succeeding order child peers, denoted as $H_{1}, \ldots, H_{M}$. However, Fig. 2 shows that the child-peers at deeper succeeding level of peer $y$ obtain only tiny gain when peer $y$ receives one additional redundant substream. The gain is defined as $\Delta Q_{m} / Q_{m}$, for example: $\Delta Q_{z} / Q_{z}$ is the gain on peer $z$ that is the first-level child-peer of peer $y$.

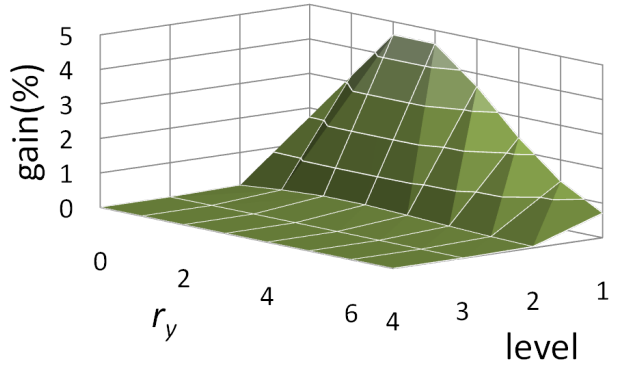

Fig. 2. Packet loss reduction for the succeeding-level child-peers of candidate peer $y$ that has received $r_{y}$ redundant substreams and request one more. $\operatorname{FEC}(24,16)$ is applied, therefore the received $r_{y}$ ranges from 0 to 7 .

Therefore, the gain on packet loss reduction to a succeeding child-peer is negligible if the depth of level is higher than one. Consequently, the energy function can be approximated well with only two terms:

$$
H^{l}(\bar{\sigma} \mid \bar{Q}, \bar{r}) \cong H_{0}(\bar{\sigma} \mid \bar{Q}, \bar{r})+H_{1}(\bar{\sigma} \mid \bar{Q}, \bar{r})
$$

The probability distribution of configuration Hamiltonian can be modeled by a Gibbs distribution [5]:

$$
P(\bar{\sigma} \mid \bar{Q}, \bar{r})=\frac{1}{Z} \exp \left[-H^{l}(\bar{\sigma} \mid \bar{Q}, \bar{r})\right]
$$

where $Z=\sum_{\bar{\sigma}} \exp \left[-H^{l}(\bar{\sigma} \mid \bar{Q}, \bar{r})\right]$ is the normalization factor.

Based on (16), the set of random variables $\bar{\sigma}$ forms a Gibbs random field (GRF). In addition, local energy is only related to the neighborhood that includes the candidate peers and its first-level child-peers. Therefore, the GRF can be represented as the Markov random field according to the Hammersley-Clifford theorem [5]. As a result, when a candidate peer obtains $y$ one additional redundant substream, the gain of packet loss reduction only propagates to its first-level child-peers. With MRF, each parentpeer can construct the optimal configuration, i.e., maximizing the probability of the configuration or minimizing the local energy function in (17), in the mesh-based P2P network.

$$
\bar{\sigma}^{*}=\underset{\bar{\sigma}}{\arg \max } P(\bar{\sigma})=\underset{\bar{\sigma}}{\arg \min } H^{l}(\bar{\sigma})
$$

\section{SIMULATION RESULTS}

We used P2Pstrsim [6] for performance evaluation. In our simulation, there are 1,000 peers in the P2P network and these peers are self-organized into a mesh structure, where the childpeers randomly select their parent-peers. The whole simulation time period is 30 minutes. The peer joining rate is 33.3 peers $/ \mathrm{min}$ and the observation period of peers is $15 \mathrm{~min}$ to $45 \mathrm{~min}$.

We encode a CIF (352x288) video at $30 \mathrm{fps}$ with bitrate 300 kbps by JM14.2 [7]. The video bitstream is protected by $F E C(24,16)$, i.e., 8 out of 24 substreams are redundant substreams. The rates of each chunk is $300 / 16=18.75 \mathrm{kbps}$.

We compare our method with the random peer selection mechanism in which parent-peers accept substream requests until their available uplink bandwidth is exhausted. To evaluate the performance of proposed method in a heterogeneous network, four different sets of channel packet loss rates were used: [0.01, 0.05], 
$[0.01,0.1],[0.01,0.15]$, and $[0.01,0.2]$. Furthermore, the peers are classified into two groups according to their uplink bandwidth: 1) $30 \%$ peers with uplink bandwidth $500 \mathrm{kbps}$, and 2) $70 \%$ peers with low uplink bandwidth. To compare the performance with different uplink capacities, we set the uplink bandwidth of class 2 to 300 kbps and $400 \mathrm{kbps}$. Consequently, the average uplink capacities of a peer in class 1 and 2 are $430 \mathrm{kbps}$ and $360 \mathrm{kbps}$, respectively.

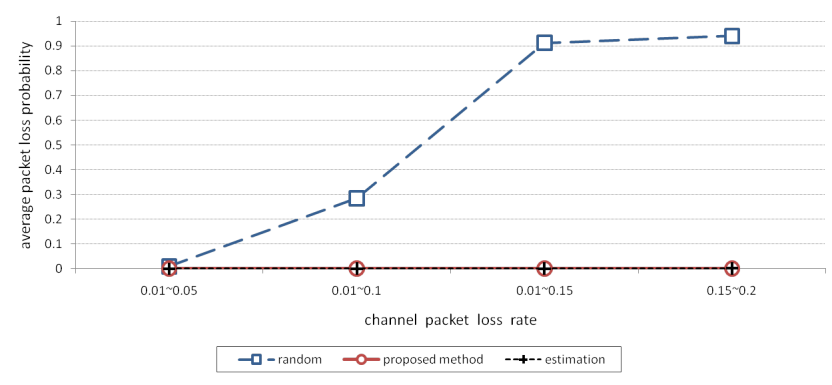

(a) The average uplink capacity: $430 \mathrm{kbps}$

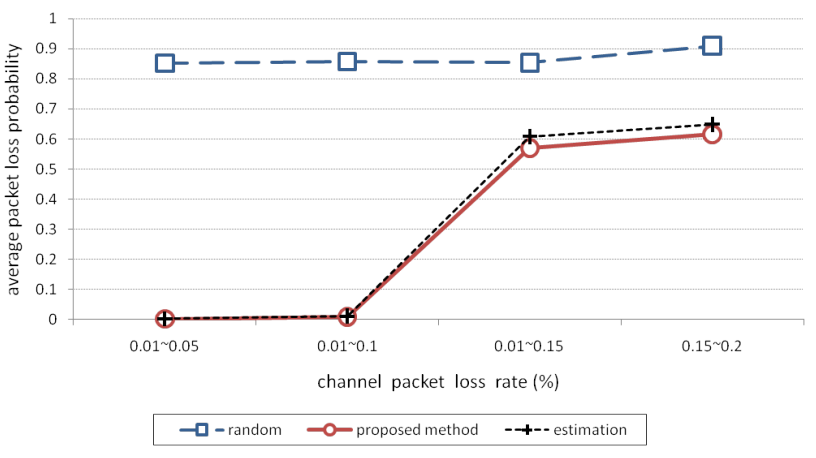

(b) The average uplink capacity: $360 \mathrm{kbps}$

Fig. 3 Average packet loss rates under different uplink capacities.

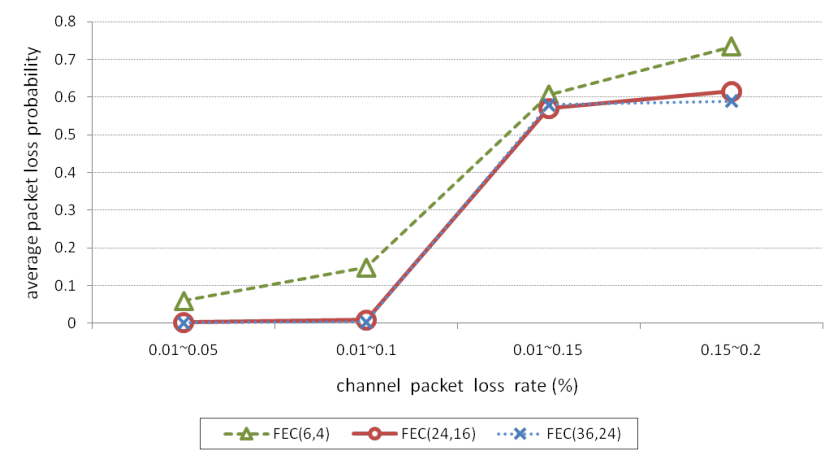

Fig. 4 Average packet loss rates with different $\operatorname{FEC}(n, k)$ codes.

Fig. 3 shows the average packet loss probability of the two methods with different uplink capacities. Fig. 3(a) shows that both methods can achieve low average packet loss probability with a low channel packet loss rate ranging within [0.01, 0.05] in a high bandwidth system. Under higher channel packet loss rates (i.e., $10 \%$ and $15 \%$ ), the proposed method still achieves low packet loss rate, 0 and $0.03 \%$, by appropriately selecting the child-peers that are likely to minimize the packet loss probability. However, the packet loss rate with random selection mechanism increases to $28.53 \%$ and $91.22 \%$, respectively. Both methods cannot recover the lost chunks under a channel packet loss rate ranging within
$[0.01,0.2]$. With a low uplink capacity, the random selection mechanism cannot recover lost packets and the average packet loss probability exceeds 0.8 in all channel packet loss settings. As shown in Fig. 3(b), the proposed mechanism still can achieve low packet loss probabilities, 0.0007 and 0.0011 , under channel packet loss rates of $[0.01,0.05]$ and $[0.01,0.1]$, respectively. The "estimation" in Fig. 3 indicates the packet loss probability estimated by the packet loss models. The results show that the packet loss models can accurately estimate the packet loss probability in different channel loss setting.

Fig. 4 shows the average packet loss rates of three different $F E C(n, k)$ codes. With the same ratio $k / n$, the packet loss rate of $F E C(24,16)$ is lower than that of $F E C(6,4)$ by about 0.088 in all channel conditions. The numbers of redundant packets, $n-k$, are 8 and 2 for $\operatorname{FEC}(24,16)$ and $\operatorname{FEC}(6,4)$, respectively. Therefore $\operatorname{FEC}(24,16)$ can provide the fine granular packet loss reduction, peers can receive the appropriate among of redundant substreams. However, the peer selection with $\operatorname{FEC}(32,24)$ does not further improve the average packet loss rate. Therefore $\operatorname{FEC}(24,16)$ provides sufficiently reliable video streaming with less channel coding overhead.

\section{CONCLUSION}

To address the packet loss problem over heterogeneous networks, we proposed a contribution-based peer selection mechanism for mesh-based P2P streaming systems. The proposed packet loss probability model considers the channel packet loss rate, peer departure, the $F E C(n, k)$ code, and packet loss propagated from parent-peers. We have shown that the packet loss propagation in a mesh-based P2P network can be modeled with Markov random field. As a result, when parent-peers select their child-peers from the candidates, only the candidates and the succeeding first-level child-peers need to be taken into consideration without introducing severe performance degradation. This drastically reduces the complexity of packet protection in mesh-based structures. Our experimental results show that the proposed method effectively mitigates the packet loss probability in a mesh-based network. Since a tree-based P2P structure is a special case of a mesh-based structure, the proposed method can provide reliable streaming services in tree-based P2P systems as well.

\section{REFERENCES}

[1] Y. Shan, I. V. Bajić, S. Kalyanaraman, and J. W. Woods, "Overlay multi-hop FEC scheme for video streaming," Signal Process.: Image Commun. vol. 20, no. 8, pp. 710-727, 2005.

[2] B. Akbari, H. R. Rabiee, M. Ghanbari, "Packet loss in peerto-peer video streaming over the Internet. Multimedia Systems. pp. 345-361, 2008 .

[3] P.-J. Wu, J.-N. Hwang, C.-N. Lee, C.-C. Gau, and H.-H. Kap "Eliminating packet loss accumulation in peer-to-peer streaming systems," IEEE Trans. Circuits Syst. Video Technol., vol. 19, no. 12, pp. 1766-1780, Dec. 2009.

[4] S. Xie, B. Li, G. Y. Keung, and X. Zhang, "Coolstreaming design, theory, and practice," IEEE Trans. Multimedia. vol. 9, no. 8, pp. 1661-1671. Dec. 2007.

[5] S. Z. Li, Markov Random Field Modeling in Computer Vision, Springer-Verlag.

[6] P2Pstrsim [online]. Available: http://media.cs.tsinghua.edu.cn/ zhangm/

[7] ISO/IEC JTC1/SC29/WG11 and ITU-T SG16 Q.6, 2007. 